See https://onlinelibrary.wiley.com/doi/abs/10.1111/papq.12253 for the final version published in Pacific Philosophical Quarterly.

\title{
Perceptual Kinds as Supervening Sortals
}

\author{
Błażej Skrzypulec
}

\begin{abstract}
It seems intuitive that in situations of perceptual recognition additional properties are represented. While much has been written about the significance of such properties for perceptual phenomenology, it is still unclear (a) what is the relation between recognitionbased properties and lower-level perceptual properties, and (b) whether it is justified to classify them as kind-properties. Relying on results in cognitive psychology, I argue that recognition-based properties (I) are irreducible, high-level properties, (II) are kind properties by virtue of being sortal properties, but (III) they supervene on lower-level properties and so are unlikely to be natural kind properties.
\end{abstract}

One of the main topics in debates regarding perceptual content concerns whether human perception represents only low-level properties, such as colors or shapes, or also highlevel properties describing the kinds of objects, causal relations, or aesthetic qualities (e.g., Bayne 2009; Siegel 2006, 2009). In the context of kind properties, some authors are willing to accept that in situations of perceptual recognition, for instance when one not only perceives an object as being big, black, and orange, but when an object also starts to look like a tiger, a new high-level property is represented. However, others claim that a change in the way an object looks, as a result of perceptual recognition, can be sufficiently explained in terms of representing additional lower-level properties.

In this paper, I analyze the kind-status of such recognition-based properties and their relation to lower-level perceptual properties. In philosophical works, recognition-based properties are usually introduced by presenting some intuitive examples like 'being a tiger' or 'being a pine tree' (Bayne 2009; Siegel 2006). However, it is not obvious how such examples should be interpreted. First, the grounds for stating that properties that are represented as a result of perceptual recognition are kind properties, are not clear. It is not sufficient to state that properties such as 'being a pine tree' allow one to classify objects as belonging to the pine-category, as the same is also true about lower-level perceptual properties that enable 
objects to be classified as belonging to categories of red things or square things (see Matthen 2005 for a theory of perceptual systems as 'sorting machines').

Second, it is not evident how such properties are related to lower-level properties. For instance, the recognition-based property of 'being a pine tree' may be identified with a proper, natural kind property concerning the evolutionary history of pine trees (see Brogaard 2013a; Silins 2013). Such natural-kind properties are independent from perceptual lower-level properties, as there may be objects of the same natural kinds with very different lower-level properties (e.g., an old pine and a pine sapling), as well as objects from different natural kinds sharing the usual, lower-level perceptual properties (e.g., a real pine and an artificial pine-like sculpture). On the other hand, a recognition-based 'being a pine tree' property may be interpreted in a radically different way, as not independent but as identical to one of the lower-level perceptual properties, for instance as identical to the shape characteristic of pine trees (Briscoe 2015; Prinz 2007).

In the paper, I argue for the two theses, SUP and RSK:

(RSK) Properties represented as a result of perceptual recognition are sortal properties and, therefore, may be interpreted as kind properties.

(SUP) Properties represented as a result of perceptual recognition supervene on lower-level properties, but lower-level properties do not supervene on R-properties.

The thesis RSK concerns the kind-status of recognition-based properties and claims that they posses such a status by being sortal properties, i.e. properties that are essential for objects, and which determine other changes that are contingent, and other changes that are essential. The thesis SUP regards the relation between recognition-based properties and lower-level properties; it states that recognition-based properties are neither identical to nor independent from lower-level properties but instead, supervene on lower-level properties. Accepting these two theses entail that recognition-based properties are high-level, kind properties, but are unlikely to be usual natural kind properties, as they are not independent from lower-level properties.

This intermediate position has several important consequences for the current debates on high-level perception. First, it goes beyond the usual dichotomy of arguing that recognition-based properties are natural kinds (in particular, biological kinds, see Brogaard 2013a; Siegel 2006; Silins 2013) versus arguing that they are identical to lower-level properties (Briscoe 2015; Nanay 2011; Pacherie 2000). I show that both these alternatives are likely to be false and that perception represents high-level 'perceptual kinds' which are not the same as biological natural kinds. Second, it is commonly claimed that if perception 
represents a property, then it provides an immediate justification that this property is instantiated, and so the debate about high-level content is relevant for the epistemological question regarding the beliefs that are justified by perceptual experiences (Siegel 2006; Silins 2013; Brogaard 2013b). According to my position, it is unlikely that perception provides an immediate justification for beliefs about natural kinds, but it justifies more than merely beliefs about lower-level properties. Third, it has been argued that the perception of natural kind properties poses a problem for naïve realists, as it is implausible that perception is able to detect such properties (Brogaard, Chomansky 2015; Cavedon-Taylor 2015). However, the argument developed in this paper shows that there may be high-level kind perception which is not a perception of natural kinds. As a consequence, proponents of naïve realism are not obliged to refute a thesis that we perceive kind properties, as perceived kind properties are not natural kind properties. Finally, it is claimed that the debate about perception of high-level properties has a relevance for the theory of consciousness, as its various resolutions entail different views about the properties that figure in a phenomenal character of experiences (Bayne 2009). Here again, my view suggests that perceptual phenomenology is not restricted to lower-level properties, but it is also implausible that it includes natural kind properties like biological kinds.

I start by specifying hypotheses about the status of properties represented as a result of perceptual recognition (section 1). Subsequently, an argument is developed to the effect that such properties are not identical to lower-level properties (section 2) and can be interpreted as kind properties, understood in terms of sortal properties (section 3). However, despite their high-level status, recognition-based properties are not independent from lowerlevel properties; rather, they supervene on lower-level properties (section 4). Finally, in the concluding section 5, I describe 'perceptual kinds' and their functions within the cognitive system.

\section{Kind properties and reducibility}

My goal here is to consider the status of properties represented as a result of perceptual recognition acts. In consequence of such an act, an object starts to look differently, as a member of some category. For instance, it starts to look like a rabbit or a tiger. Many authors believe that this change in the way an object looks concerns a phenomenal look (e.g., Price 2009). However, there is no agreement on the type of phenomenology that is changing during perceptual recognition. It is claimed that a perceptual recognition results in an 
experience with a different sensory phenomenology (Bayne 2009; Siegel 2006), a different quasi-sensory/quasi-cognitive phenomenology (Reiland 2014) ${ }^{1}$, or if there is any phenomenal change at all, it is only a change in cognitive phenomenology connected with forming a 'perceptual belief' (Lyons 2005ab). Alternatively, a difference in the way an object looks is characterized not in phenomenal terms, but by a reference to perceptual mechanisms (see Fish 2013; Lyons 2005b). In this perspective, a perceptual recognition is a form of recognition, such that the resulting look of an object is an output of perceptual mechanisms. Because perceptual recognition plausibly involves the application of concepts, the relevant perceptual mechanisms are those constituting high-level perception (Lyons 2005b; Reiland 2015). According to psychological models, such mechanisms are responsible for perceptual categorization and involve comparing the current input with patterns stored in the long-term memory (Hummel, Biederman 1992; Hummel 2013; Ullman 1996). The most popular behavioral indicator of the fact that a property can be perceptually recognized is a short time needed for recognition (Bayne 2016; Fish 2013).

Despite the above differences, authors agree that acts of perceptual recognition must be distinguished from acts of cognitive recognition, which can be based on perceived properties. Such acts of cognitive recognition consist in forming non-perceptual beliefs, in which properties are attributed to objects by relying on the content of perceptual experiences. Quite obviously, virtually any property, including natural kinds properties, can be attributed as a result of a cognitive recognition, therefore it is trivial that high-level properties can be represented in such a way (Cavedon-Taylor 2015; Prinz 2013). From the point of view of perceptual processing, acts of cognitive recognition may modify the way things look, but this difference results from the functioning of post-perceptual mechanisms. As it is sometimes stated, they modify the way things epistemically look or seem (Brogaard 2013b; Fish 2013). An important feature that distinguishes perceptual recognition from cognitive recognition, is that effects of perceptual recognition are largely independent from background knowledge (Bayne 2009; Block 2014; Reiland 2015). For instance, an object can be perceptually recognized as a rabbit, and it still looks like a rabbit even if one learns that, in fact, it is a hologram (Reiland 2014; Siegel 2006). Similarly, a rubber toy can be perceptually categorized as a chicken, regardless of any possessed knowledge about its toy status (Lyons 2005b). On the other hand, recognizing a transparent liquid as $\mathrm{H}_{2} \mathrm{O}$ seems to be an example of a cognitive recognition. After learning that, in fact, it is XYZ, an experience changes, as the liquid no longer epistemically looks like $\mathrm{H}_{2} \mathrm{O}$. 
In this paper, I consider perceptual recognition from the point of view of perceptual processing, as this perspective allows for a broader use of psychological data concerning recognition-related phenomena. Thus, acts of perceptual recognition can be characterized as mental acts that result in an object looking as a member of some category, and this look (a) is an output of high-level perceptual mechanisms, and (b) is largely independent from background knowledge.

Plausible examples of perceptual recognition are associated with perceptual learning. For instance, without training, people are usually unable to differentiate between house sparrows and tree sparrows. However, with practice, their high-level visual mechanisms are more sensitive to relevant differences, which allows for rapid recognition. The classical philosophical example concerns a person who learns to recognize pine trees and as a result pine trees start to look different to her (Siegel 2006). As a result of perceptual learning, perceptual categorization mechanisms form a new, stable pine-pattern, stored in long-term memory, and are able to match this pattern with the currently available stimuli. When a person perceives a pine tree, the pine-pattern is matched with the input and in consequence the object starts to look like a pine tree, a process that may result in forming a perceptual belief, 'This is a pine tree.' A pine tree look is likely to be present even in a situation in which a person has additional knowledge suggesting that what is perceived is not a real pine tree.

It seems plausible that a change in the way an object looks that is associated with an act of perceptual recognition indicates that an additional property is represented. Such properties are often intuitively referred to by using natural kind names. However, the mere act of introspection is not sufficient to determine the status of such properties, and many authors believe that they should be identified with lower-level properties.

The above characterization of perceptual recognition is a broad one, and abstracts from the controversies concerning the nature of phenomenal change that may be associated with perceptual recognition. My main goal is not to decide what kind, if any, of phenomenal change results from perceptual recognition, but to investigate the nature of properties represented as a consequence of such acts. These issues are largely independent, since even if perceptual recognition involves a change in quasi-sensory/quasi-cognitive phenomenology, and not in pure sensory phenomenology, it may still be the case that this non-sensory phenomenal change occurs merely by representing some additional lower-level properties. Later, I use the term 'R-properties' to designate properties that are represented as a result of perceptual recognition acts, and I discuss their low- or high-level status by analyzing psychological data concerning perceptual categorization. 
One important way of arguing within the discussion concerning R-properties involves constructing and analyzing phenomenal contrast cases, i.e. cases comparing situations in which a person has mental states with different phenomenal characters (Nanay 2011; Prinz 2013; Siegel 2006). While some authors believe that analysis of contrast cases does not offer a reliable guide to the low- or high-level status of R-properties, and that the debate should be more focused on interpreting scientific data (e.g., Fish 2013) —which is also the main methodology applied in this paper-referring to contrast cases still plays a useful heuristic role in showing the difference between competing positions. The majority of authors who analyze contrast cases believe that acts of perceptual recognition modify the phenomenal character of experiences. Relying on this assumption, the question of the best explanation for this phenomenal change related to perceptual recognition is discussed. One popular hypothesis is that phenomenal difference arises due to representing a kind property that is irreducible to lower-level properties (Bayne 2009; Siegel 2006) ${ }^{2}$. On the other hand, it is a common claim that the R-property responsible for change in phenomenal character is identical to one of the lower-level properties like those connected with size, color, spatial position, and shape (Briscoe 2015; Connolly 2014; Jagnow 2015).

In fact, the above two popular positions combine two largely separate problems. The first is whether R-properties are kind properties. The second concerns the reducibility of Rproperties to lower-level properties. The usual solutions combine being a kind property with irreducibility and reducibility with not being a kind property. However, there is nothing inconsistent in claiming that R-properties are not kind properties but some other high-level, irreducible properties. Analogously, one can maintain that there are some kind properties that are identical with lower-level properties. In what follows, I will treat these two problems separately.

While there is a strong intuition that properties like 'being a tiger' or 'being a pine tree' are kind properties, while 'being red' or 'being square' are not, the criterion for this distinction is less obvious. Justifying the difference between kind properties and other properties is especially difficult in the case of perception, as every perceptual representation may be interpreted as involving a classification (see Matthen 2005: 22-35). In virtue of representing lower-level properties, objects are classified as belonging to categories of red or square things, and as a result of perceptual recognition acts they are classified as belonging to categories of tigers or pine trees.

A more promising idea is to distinguish kind properties by referring to the notion of sortal properties. According to both philosophical and psychological characterizations, sortal 
properties are those that satisfy two conditions: (1) they are essential for the sameness of objects that posses them and (2) they determine which other changes are contingent and which are essential (Wiggins 2001: 55-58; Carey, Xu 1999) ${ }^{3}$. For instance, classifying an object as a red thing does not provide much information about the changes which preserve and the changes which break the identity of an object. On the other hand, categorizing an object as a dog allows for various inferences concerning its sameness through time. For example, seeing a small dog now and a larger dog at a later time does not justify the claim that these objects are different, as growth is a common pattern of a dog's development. The sortal property of being a dog determines that this change is contingent. However, classifying an object $\mathrm{X}$ as a dog with a property of being a Yorkshire Terrier gives a strong reason to think that no object with a property of being a Doberman should be identified with $\mathrm{X}$, as such change would be essential for dogs. In addition, the property of being a dog is essential itself, therefore an object classified as a dog cannot be identified with an object classified as a porcupine.

In this paper, kind properties will be understood as sortal properties, and I will investigate whether representing objects as having R-properties determines the changes that are essential and those that are contingent. More specifically, I consider the following hypothesis $R S K$ :

(RSK) Properties represented as a result of perceptual recognition are sortal properties and, therefore, may be interpreted as kind properties.

In section 3, I offer arguments in favor of the hypothesis $R S K$.

The second, independent problem concerns the relation between R-properties and lower-level properties. According to a reductive proposal, R-properties are identical to some perceptual lower-level properties and so the following hypothesis is endorsed:

(RED) R-properties are identical to lower-level properties.

The most popular reductive proposition is to identify R-properties with so-called 'shape-gestalts,' shapes represented as a result of attentional processing (Prinz 2013). As an illustration, let's consider again Sigel's classic example of a person who has gained the perceptual ability to recognize pine trees (Siegel 2006). To the hypothetical person, pine trees look different before and after gaining this new ability. Nevertheless, proponents of a reductive approach to R-properties claim that this difference is sufficiently explained by the fact that gaining recognition ability changes the represented shape property. When a person learns to perceptually recognize pine trees, she starts to focus attention on different parts of a tree. By virtue of these variations, some elements of shape are represented in a more detailed 
way, while others, which are outside the focus of attention, receive only a rudimentary representation (Briscoe 2015; Jagnow 2015; Pacherie 2000; Price 2009; Prinz 2007).

In the beginning of this section, I have noted that acts of perceptual recognition are likely to be realized by top-down perceptual mechanisms that compare the current input with patterns stored in memory. One may suppose that this is enough to falsify RED as properties represented in virtue of functioning of top-down mechanisms are high-level properties. However, it is not the case as nothing prevents properties represented in a top-down manner from being properties that are considered as lower-level within the philosophical debate concerning high- and low-level perception (see Brogaard, Chomanski 2015). For instance, it might be the case that top-down categorization mechanisms described in psychological models are those that allow representation of gestalt-shape properties which are considered as lower-level in philosophical discussions.

Authors who reject a reductive approach to R-properties often characterize them by giving examples of natural, especially biological, kinds (Bayne 2009; Siegel 2006). Such natural kind properties are not only irreducible to lower-level properties, but are independent from them. This means that they neither supervene on lower-level properties and nor do lower-level properties supervene on them. Lower-level properties may change without a change of a natural kind property, as when a pine tree develops from a sapling to an old tree. Analogously, a natural kind property may be lost despite the preservation of perceptually represented lower-level properties, as in the transition between a living and a stuffed animal. If R-properties are such natural kind properties, then the hypothesis that R-properties are identical to lower-level properties is false and instead the following statement should be accepted:

(IND) R-properties are independent from lower-level properties.

Again, it should be noted that while R-properties are likely to be represented by topdown mechanisms, this fact is not sufficient to prove $I N D$, just as it is insufficient to falsify that R-properties are identical to lower-level properties.

In sections 2 and 4, I argue that R-properties are neither identical to nor independent from lower-level properties because R-properties supervene on lower-level properties, but lower-level properties do not supervene on R-properties (and so they cannot be identical to Rproperties). Instead, I show that the following SUP hypothesis is true:

(SUP) R-properties supervene on lower-level properties, but lower-level properties do not supervene on R-properties. 
Joint acceptance of the sortal-status of R-properties and SUP leads to the conclusion that R-properties are kind properties (understood as sortal properties) that supervene on lower-level properties. In the subsequent sections, I build my arguments by relying on psychological data which plausibly concern cases of perceptual recognition. This is done by constructing 'recognition contrast cases', through which I identify situations, with a special focus on experimental ones, that differ in terms of performed acts of perceptual recognition. I argue that by investigating such cases it can be claimed that R-properties supervene on lowerlevel properties, and also that they are sortal properties.

\section{The irreducibility of R-properties}

In this section, I argue against the hypothesis that R-properties are identical to lowerlevel properties. If R-properties are identical to lower-level properties, then for each Rproperty there is a single lower-level property, such that representing this lower-level property is necessary and sufficient for representing the considered R-property. In other words, for each R-property an object can look as having this property if and only if it looks as having a certain lower-level property. However, it seems that this condition is not satisfied.

The claim that representing a lower-level property is not necessary for representing an R-property can be justified by referring to recognition contrast cases. A relevant contrast case would be constituted by two situations $S 1$ and $S 2$, such that (a) in both, a person represents the same R-property by performing an act of perceptual recognition, but (b) in $S 1$, a perceptual recognition act occurs in virtue of representing a lower-level property $F$, and in $S 2$ in virtue of representing a different lower-level property $G$. In this case, the R-property represented in $S 1$ and $S 2$ cannot be identical with the property $F$, as this property is not represented in $S 2$; it cannot be identical with $G$, as this property is not represented in $S 1$; and it cannot be identical with both $F$ and $G$, as it would entail that these two different properties are identical. The occurrence of such contrast cases concerning R-properties would falsify the hypothesis that R-properties are identical to lower-level properties. Using the language of looks, such a result would mean that things can look as having the same R-property despite the significant changes concerning their lower-level properties.

According to the most popular reductive position, R-properties may be identified with shape-gestalt properties, i.e. shape properties represented as a result of attentional processing (Briscoe 2015; Pacherie 2000; Price 2009; Prinz 2013). However, it is likely that the same Rproperty may be represented in virtue of representing several different shape-gestalt 
properties. This is because an object can be perceptually recognized from different viewpoints, which may significantly differ in terms of accessible shape properties. It is commonly accepted within cognitive psychology that mechanisms of perceptual categorisation can ascribe an object to the same category despite differences in the perspective from which an object is observed (Hummel, Biederman 1992; Ullman 1996). While shapegestalts are likely to capture characteristics of shapes that are invariant across many views (Hummel 2013), so there is no need to represent a different shape-gestalt for each viewpoint, there are still viewpoints that are so different that it is unlikely that we could represent the same shape-gestalts in each of them. For instance, one may recognize something as a wolf when seeing a wolf from the side, as well as when seeing a front of a wolf's head. In these two situations one perceptually recognizes a thing as a wolf, while the represented shapegestalts seem to be very different.

The above example is likely to be true even if human vision is able to represent 3dimensional shape-gestalts. Such 3-dimensional shapes can be understood as conjunctions of shape-properties indexed by perspectives from which they are visible, like being $\mathrm{F}$ from the front-perspective and being $\mathrm{G}$ from the side-perspective. One may suppose that when seeing a wolf's head and seeing a wolf from the side we are representing the same 3-dimensional shape. However, because not all shape-properties of an object are perceived at once, to represent a 3-dimensional shape, the shape properties associated with various perspectives have to be somehow inferred from the currently perceived shape-properties. In some simple cases the currently perceived shape-properties provide reliable cues of how the shape would change after rotation. For instance, from seeing a cube such that one of its edges is directly in front of us, we can predict how a cube will look if one of its sides would be in front of us. However, there is no simple geometrical transformation that would allow us to predict how a wolf looks from a side-perspective after perceiving the front of its head. It is more plausible that if a 3-dimensional wolf-shape is represented as a result of representing a wolf's headshape, then this transition is already mediated by recognizing an object as a wolf. The sequence would be: (a) a wolf's head-shape is represented, (b) relying on that, it is represented that an object is a wolf, (c) this recognition allows us to predict how the object looks from other views, and (d) as a result a 3-dimensional shape is represented. In consequence, a wolf can be perceptually recognized by representing various local shapegestalts, and each of these recognitions allow for a further representation of a holistic 3dimensional shape-gestalt. 
What is more, there are empirical data that show that acts of perceptual recognition do not always happen solely in virtue of representing shape-related properties. One reason for this is that perceptual recognition is context-dependent. In particular, objects are recognized more quickly when they are perceived in the usual surroundings for objects of a given kind, or when they have typical properties different to those concerning shape (for instance, a characteristic location within a scene, see Fenske et al. 2006 for a review). The fact that the time needed for recognizing an object depends on context allows for formulating a relevant recognition contrast case. Let's assume that an object $A$ is recognized after time $T$ if presented without any contextual cues, so it is likely that this recognition happens in virtue of attentively processing its shape. However, when $A$ is given in an appropriate context, the recognition time is shorter than $T$. This suggests that in the second case, an act of a perceptual recognition was performed before the time that is needed for representing a shape-gestalt has passed. In such a case, the object $A$ was recognized without relying on a represented shape-gestalt but probably in virtue of forming some less elaborated shape-representation, together with representing other properties related to the context of the scene.

Moreover, at least some R-properties can be represented even if the information regarding shape is largely unavailable. For example, people are able to differentiate males and females relying on patterns of walking motion when the overall shape is invisible and the only source of information is lights attached to body-joints (Bullot, Rysiew 2007: 287). Once again, this shows that acts of perceptual recognition resulting in representing the same Rproperty may rely on representing different lower-level properties.

In fact, in psychological works concerning perceptual recognition, it is claimed that the human perceptual system may adapt different strategies for recognizing objects depending on the task's demands (see Ullman 1996 for a classical review of different variants). For example, in an easy task involving recognizing a letter $B$ among other letters or a banana among other fruits, there is no need to process the whole shape, as recognition may happen relying or recognizing some set of diagnostic features, like the presence of two holes in case of $B$ or the presence of yellowness and being curved in the case of a banana. On the other hand, harder tasks require not only representing a shape-gestalt but also performing a mental rotation to match the currently represented shape with a prototype stored in long-term memory (Edelman, Bülthoff 1992). Again, the presence of such task-dependence variations in abilities for perceptual recognition serve as an argument that there may be recognition contrast cases involving representing the same R-property in virtue of representing different lower-level properties. 
The provided empirical evidence can be plausibly treated as concerning perceptual rather than merely cognitive recognition, as it concerns the functioning of perceptual categorization mechanisms, and in many of the provided examples recognition can happen rapidly. In consequence, the above considerations show that while R-properties always seem to be represented as a result of representing lower-level properties, a single R-property can be represented in virtue of representing various lower-level properties depending on task, context, available perceptual information, time, and cognitive resources. Hence, it is unlikely that an R-property would be associated with a single lower-level property that is necessary and sufficient for representing this R-property, so there is no lower-low level property with which it can be identified.

One can still maintain that while an R-property cannot be identified with a single lower-level property or a combination of lower-level properties, it can be identified with a complex disjunction of lower-level properties. I do not argue against this position because I believe that disjunctions of lower-level properties, if they are in fact perceptually represented, are not lower-level but high-level properties. First, disjunctions of lower-level properties do not figure as examples of lower-level properties, unlike colors, shapes, or locations, discussed in philosophical works (e.g., Bayne 2009; Brogaard 2013a; Siegel 2006). Second, and more importantly, to ascribe a property identical to a disjunction of lower-level properties to an object it is not enough, in contrast to the usual examples of lower-level properties including shape-gestalts, to process the current perceptual input. This is because such a disjunction can contain operands that do not correspond to the properties of a currently perceived object and so complete information about the content of a disjunction cannot be obtained from the stimuli. For instance, a disjunction 'red or square' can be ascribed to a green square when the input does not contain information about redness. Because of this, representing properties that are disjunctions of lower-level properties requires using some additional information, which makes it less plausible to interpret them as lower-level properties.

\section{R-properties as sortal properties}

In the above section, I have argued that R-properties are not identical to lower-level properties. Below, I show that they should also be understood as kind properties. According to the hypothesis $R S K$, R-properties are kind properties due to being sortal properties, i.e. properties that are essential for an object, and that determine the changes that are essential and those that are contingent. Once again, this hypothesis can be tested by investigating the 
occurrence of certain recognition contrast cases. The hypothesis that R-properties are sortal properties will be justified if there are situations $S 1$ and $S 2$ such that (a) in $S 1$ and $S 2$ a person perceives an object, and in $S 1$ this object is perceptually recognized as having an R-property, but this is not the case about an object in $S 2$; and (b) the identity of an object in $S 1$ is represented differently to the identity of an object in $S 2$, because in $S 1$ the R-property is essential, and some changes that are essential for objects without the R-property are contingent for an object with the considered R-property or vice versa; and (d) the most plausible explanation of differences in representing the identity of objects in $S 1$ and $S 2$ is that only the object in $S 1$ is represented as having the R-property.

I argue in favor of the hypothesis that R-properties are sortal properties by referring to four types of psychological studies concerning how objects' diachronic identity is perceptually represented. The first type of study investigates apparent motion, i.e. the phenomenon that successively presented objects may be perceived as a single entity. The second justification comes from studies operating within the Multiple Object Tracking Paradigm, where participants simultaneously track several objects among distractors. The third example concerns priming studies, which investigates how multiple presentations of the same stimuli influence the time needed for recognizing it. Finally, I refer to research in developmental psychology whose topic is infants' identity perception.

\subsection{Apparent motion}

One of the best-known examples of apparent motion is the phi-phenomenon (see Matthen 2004). When one is presented with two light spots, alternatively appearing and disappearing, one can perceive a single moving object or two blinking objects depending on the distance between spots and the temporal relation between the disappearance of the first and the appearance of the second. In fact, the spatiotemporal relations are considered crucial for determining whether successively presented objects are perceived as a single entity; and usually an object is identified with the closest successive object even if more similar but also more distant objects are also presented (see Nakayama et al. 1995 for a review).

However, this is not universally the case, and exceptions are connected with objects' perceptual recognition. It has been shown that if participants are presented with successive pictures of people moving certain body parts, the apparent movement of these parts is not perceived along the shortest route but along a route that is consistent with actual human movement-abilities (Shiffrar, Freyd 1990). For instance, an arm cannot move through the rest 
of the body, and so such a line of movement is not perceived. Later, this result was extended for other categories of objects that are not perceived as moving through a solid wall, even if this route is the shortest one (Solberg, Brown 2002). Similarly, parts of an object are perceived to move differently when they are components of a rotating recognizable figure than when they constitute a rotating unfamiliar composition (Ramachandran et al. 1998). When a figure is recognizable, its parts do not appear to move following the shortest route, as in the case of an unfamiliar composition, but in a way that preserves the role that a given part serves within the figure. For instance, in case of a face-shaped figure, the apparent movement combines a nose-part from an initial presentation with a nose-part from a figure after rotation.

Such examples suggest the existence of situations that constitute a relevant contrast case in favor of the hypothesis that R-properties are sortal properties. First, there are situations in which (a) a person perceives an object presented earlier as diachronically identical to an object presented later; (b) these objects are visually connected by a movement along a shortest route; and (c) when perceiving these objects the person does not represent these objects as having R-properties. Second, there exist situations where (a) a person perceives an object presented earlier as diachronically identical to an object presented later; (b) these objects are visually connected by a movement along a route different from the shortest one; and (c) when perceiving these objects the person recognizes them as having an R-property.

The change of the perceived movement-route is related to a difference in the identity conditions of objects. If diachronically identical objects are not connected by the shortest route, then there is something that makes this route unavailable for these objects. In other words, there is something about these objects that makes undergoing some changes unlikely while maintaining diachronic sameness. Because the shortest movement-route becomes unavailable when objects are represented as having R-properties, while other variations in objects' features do not have such influence on apparent movement, it seems that the modification of identity conditions happens as a result of ascribing R-properties. The Rproperties impose restrictions on the way an object can change without breaking its identity, so some changes that are contingent without R-properties become essential as a result of perceptual recognition. Such ability serves as a justification for interpreting R-properties as sortal properties.

\subsection{Multiple Object Tracking}


In Multiple Object Tracking (MOT) experiments, people are presented with some number of randomly moving objects and are asked to track some of them while the remaining ones serve as distractors (Pylyshyn 2007: 34-37). At the end of the experiment, the objects stop moving and participants point out which objects are targets. One of the main sources of identification-errors in MOT experiments is swapping the objects' identities when a target and a distractor move close to each other (Drew et al. 2013). This can lead to an ambiguous situation in which there are two objects at a moment $\mathrm{T} 1$ and two object at a subsequent moment $\mathrm{T} 2$ such that both of the objects at T2 are possible candidates for being identified with a target from T1. Such a situation may lead to a mistake in tracking, as a target from T1 can be identified with a distractor from T2. In a standard version of MOT, all presented objects are qualitatively the same (e.g., black circles of the same size). However, there is a version of MOT, known as Multiple Identity Tracking, in which all the presented objects have different features (Oksama, Hyona 2008). In this case, to avoid making the task trivial, objects hide behind occluders when their movement stops and participants choose those occluders that correspond to targets.

A Multiple Identity Tracking experiment that is directly relevant to considerations regarding R-properties has been conducted by Wei and colleagues (Wei et al. 2016). The study concerns how the error-rate changes when targets and distractors belong to different categories (such as mammal, bird, fruit, furniture). It has been revealed that the participants are significantly more successful when there is a categorical difference between targets and distractors, e.g. each target is a different mammal and each distractor a different bird, compared to cases where all objects are of the same category (e.g., each object is a different bird). Moreover, the effect disappeared when objects were presented upside-down, which makes recognition more effortful while not modifying other differences between objects. This suggests that the difference in error-rate was indeed caused by a difference in category and not only due to differences in features between objects belonging to different categories. The lower number of errors when targets and distractors belong to different categories suggests that ambiguous situations in which a target is in proximity to a distractor are easier for the visual system to solve if both objects are perceptually recognized as categorically different.

The above result constitutes another piece of evidence for the hypothesis that $\mathrm{R}$ properties are sortal properties. It allows for formulating a contrast case that compares: (1) a class of ambiguous situations where a target is in proximity to a distractor, and each of these objects is likely to be recognized as having a different R-property; and (2) a class of ambiguous situations where a target is in proximity to a distractor, and objects are not 
recognised as having $\mathrm{R}$-properties, or the same R-property is ascribed to both objects. The lower error-rate in class (1) cannot be explained solely in terms of differences in lower-level properties, as demonstrated by experiments with objects presented upside-down. However, it can be plausibly explained by an influence of R-properties on identity conditions. According to this interpretation, the visual system is less likely to identify objects that differ in Rproperties than those that differ only in lower-level properties, hence ambiguous target/distractor situations are resolved more easily in the case of class (1). Ascribing an Rproperty to an object modifies its identity conditions, as objects that do not have this Rproperty are very unlikely to be identified with the considered object. This suggests that the R-properties are essential for objects.

\subsection{Priming studies}

It is well known that a feature is recognized faster when it is presented again shortly after the first presentation (the so-called nonspecific preview effect). What is more, this effect is even stronger if a feature appears on the same object as before (the so-called object-specific preview effect, Gordon, Irwin 1996). While in psychological works concerning priming effects the presented elements are usually called 'features', in the majority of studies, these 'features' are letters, words, or geometrical figures and so they may be interpreted as objects (Gordon, Irwin 2000; Kahneman et al. 1992; Moore et al. 2010). Under this interpretation, priming studies can tell us something about the conditions that determine that an object presented later is recognized as the same as one presented earlier.

The psychological experiments reveal that when recognizable objects are presented, like letters or words, then priming effects occur even if during the second presentation the properties of the objects are different. In particular, priming effects appear if letters or words are written in a different case in each of the presentations (Gordon, Irwin 1996; 2000). This suggests that such familiar objects are treated as being the same even when some significant changes concerning their shape have occurred. However, when successively presented objects are not familiar letters or words but some novel figures, the priming effects are not present when figures in successive presentations differ in shape (see Moore et al. 2010 where ornaments were used instead of Latin letters).

Once again, relying on such results, we may formulate a contrast case composed of two situations: (1) an object is recognized as having an R-property, and its successive presentations invoke priming effects despite changes in shape; and (2) an object is not 
recognized as having an R-property, and priming effects do not appear when a subsequently presented object has a different shape. Because the presence of priming effects is interpreted as a result of representing an element to be the same, the above difference in situations composing a contrast case can be explained by postulating that representing R-properties modify identity conditions. While in examples concerning apparent motion and MOT some changes were unavailable for objects with R-properties, here, in virtue of representing Rproperties, some changes, which break the identity of unfamiliar objects, are treated as contingent. In other words, some essential changes become contingent as a result of representing an R-property.

\subsection{Infants studies}

Studies on how infants represent identity between objects rely on measuring how long infants look at the outcome of the presentation. This allows us to distinguish which outcomes are expected and unexpected for an infant. For instance, an infant may be presented with two spatially separated curtains. A ball appears from behind of one of the curtains and then hides again. Subsequently, the same happens with the second curtain. Then the curtains are removed and (1) only one ball is visible or (2) two balls are visible. Outcome (1) draws infants' attention for a longer time and is interpreted as being unexpected. Such a result has lead researchers to believe that infants perceive objects as being distinct if there is no spatiotemporal continuity between them (Carey, Xu 2001).

By using the above paradigm it was observed that until they reach 12 months, infants do not treat featural changes as indicating a lack of identity between objects $(\mathrm{Xu}$, Carey 1996). This was shown using a presentation containing a single curtain. At first an object appears from behind a curtain and then hides again. Subsequently, an object with different features appears and hides. When this design is used, infants do not treat an outcome with only one object as unexpected. This result was additionally confirmed by studies concerning infants' exploratory behaviours (Van De Walle et al. 2000). When infants see the following pattern of events: a toy is drawn from a box and put back again; later a toy with different features is drawn from a box and put back again; the infant searches for a single item in the box.

The situation changes when infants reach the second year of life. Then objects start to be treated as numerically different when they belong to different categories (Xu 2007). For instance, when a duck-toy and a car-toy alternatively appear from behind the curtain and then 
hide, the outcome with only one object is unexpected. However, the one-object outcome is not treated as unexpected when alternatively presented objects differ in features concerning color, size, and shape that do not involve a categorical difference (Xu et al. 2004).

This result shows that in the relatively early stage of development contrast cases can be found, which suggests that representing R-properties determine that objects belonging to different categories should not be identified with each other. First, there are situations in which infants perceive objects, like a duck-toy and a car-toy, that are likely be recognized as having R-properties. These objects are represented as being distinct, as the outcome the presence of a single object is treated as unexpected. Second, in cases where infants perceive objects that do not belong to any common categories or which belong to the same category but which differ in other properties, the outcome with a single object is not treated as unexpected. This suggests that non-categorical differences do not entail a representation of two numerically different objects. Such differences in identity conditions introduced by representing R-properties corroborates the hypothesis that R-properties are sortal properties by showing that within the developing cognitive architecture R-properties are essential for diachronic identity.

\subsection{Summary}

All the above results, coming from different experimental paradigms, suggest that representing an object as having an R-property determines identity conditions in a way that corroborates the sortal status of R-properties. First, MOT studies and infants studies suggest that R-properties are themselves essential as objects with different R-properties are unlikely to be identified with each other. Second, apparent motion and priming studies show that as a result of ascribing R-properties some contingent changes become essential while some essential changes may become contingent. These two above characteristics are defining features of sortal properties as defined in section 1. In consequence, R-properties can be treated as sortal properties, and in this sense as kind properties.

\section{Dependency of R-properties}

In the previous sections, I argued that R-properties cannot be identified with lowerlevel properties and that they are kind properties. Here, I will show that despite their highlevel status, R-properties are not independent from lower-level properties. 
If R-properties are independent from lower-level properties, then R-properties do not supervene on lower-level properties and lower-level properties do not supervene on them. Supervenience here is understood in the usual way: $A$ supervenes on $B$ if and only if every change concerning $A$ entails a change concerning $B$. From the section 2, it is clear that lowerlevel properties do not supervene on R-properties because the same R-property may be represented in virtue of representing different low-level properties. As a result, there are possible changes of lower-level properties that do not entail a change of R-property. However, the crucial question is whether R-properties supervene on lower-level properties.

The hypothesis that R-properties do not supervene on lower-level properties can be justified by offering contrast cases composed of situations $S 1$ and $S 2$ such that (a) in $S 1$ a person correctly represents an R-property $R l$ in virtue of representing some lower-level properties; and (b) in $S 2$ a person starts to correctly represent a different R-property, $R 2$ instead of $R 1$, without any change in represented lower-level properties. The correctness condition is needed because it is obvious that virtually any R-property can be represented in virtue of representing any lower-level property given the occurrence of some errors. I believe that such contrast cases cannot be found when R-properties are concerned, and so Rproperties supervene on lower-level properties.

It should be noted that if perceptual recognition happens in virtue of mechanisms that compare the current input with memorized patterns, then the lack of supervenience of Rproperties on lower-level properties can happen only in special circumstances. It requires that a perceptual system has learned two patterns, $P 1$ and $P 2$, which despite being different can be applied to the exactly the same set of represented lower-level properties $F$. To make it possible, patterns $P 1$ and $P 2$ must possess a disjunctive structure with a common element $F$, for instance ' $F$ or $G$ ' in the case of $P 1$ and ' $F$ or $K$ ' in the case of $P 2$, as then they can be different, yet both applicable to F. Furthermore, there must be a factor unrelated to changes in lower-level properties that determines which of the patterns $P 1$ and $P 2$ is applied when $F$ is represented. Given that these requirements are very specific, and that to successfully distinguish objects from multiple categories, perceptual systems need to minimize overlaps between memorized patterns, it seems plausible to assume that in case of the majority of common R-properties there is a supervenience between them and lower-level properties.

Nevertheless, below, I argue that even if we consider some special cases, in which Rproperties may be prone to change without lower-level changes, there is still no strong evidence for the lack of supervenience. To investigate these cases let's consider the factors that may change the represented R-properties without changing represented lower-level 
properties. One possibility is that there are cases of 'primitive categorization' in which perceptual categorization is simply a matter of matching a long-term memory representation with an input, without any other changes. A second option is that the represented R-property may change due to the influence of some propositional factors. Third, it may be the case that such changes happen solely as a result of temporal factors, for instance being connected with previously presented stimuli. Finally, one may propose that R-properties do not change due to lower-level changes, but as a result of changes in other types of perceptually represented highlevel properties.

\subsection{Primitive categorization}

According to psychological models, perceptual categorization happens when a current stimuli is matched with a pattern that is stored in a long-term memory. The simplest patterns may be in the form of a set of features which are diagnostic for a members of a given category (see Ullman 1996). Relying on such psychological background, one may propose that in some cases, perceptual recognition consists solely of matching elements of a pattern with elements of a perceived object, without any modification in the represented lower-level properties of this object. In particular, such intuition may arise from considering cases of perceptual learning, in which a new pattern is formed, relying on the observed similarities in the perceived objects. As a result of perceptual learning, a represented R-property changes. Before creating a new pattern, there are objects which are not represented as having a certain R-property, but after learning, they are represented as having this property. Due to the fact that perceptual learning is not usually associated with an obvious phenomenal change that would suggest a modification of represented lower-level properties, it may be believed that it involves cases of primitive categorization.

Nevertheless, there are serious reasons to believe that in such cases of perceptual learning, representing a new R-property involves a modification of represented lower-level properties. Even the simplest form of perceptual learning has to rely on making a distinction between properties that are important for belonging to a given category, and those which should be neglected, as they reflect only intra-category variations. Hence, making a perceptual recognition by using a learned pattern is likely to involve enhanced processing of relevant properties, as well as inhibiting unimportant ones that may be distracting. As a result, perceptual learning causes some properties to be represented in a more detailed way, while others obtain a more rudimentary representation. 
The above theoretical hypothesis gains empirical support from the experiments concerning perceptual recognition of novel categories (e.g., various blob-shaped things). It has been observed that after a period of learning, participants have greater skills in distinguishing the diagnostic features of objects, when an object from a newly learned category is compared with an item of a different type (Goldstone 1994; Schyns, Rodet 1997). For instance, before learning, people may be unable to distinguish that two objects have slightly different shapes, $S$ and $S^{\prime}$. However, if shape $S^{\prime}$ is a diagnostic property of an artificial category $\mathrm{C}$, and participants learn to recognize members of this category, then they are more likely to classify shape S', possessed by members of C, as different from the shape of objects having S. It seems that before perceptual learning, objects having S and S' are represented as possessing the same lower-level shape-property, but after learning, the represented lower-level properties change, and exemplars of $\mathrm{C}$ having $\mathrm{S}$ ' are assigned a new shape-property. Analogously, after a period of learning, the ability to distinguish nondiagnostic features become suppressed. Let's assume that having an exact size is contingent for members of a newly learned category $\mathrm{C}$. In such a case, perceptual learning may cause a person, who before learning was able to distinguish between slightly different sizes of objects, to perceive such sizes as being the same, when a size of an exemplar of $\mathrm{C}$, and a size of some other item are compared. Again, it suggests that perceptual recognition changes the represented lower-level properties. Properties which were represented as different before recognition, are represented as identical after learning a novel category. In addition, it has been observed that perceptual learning of novel categories changes the way in which objects are divided into parts (Schyns, Murphy 1994).

The effects of perceptual learning are restricted to comparing the members of a newly learned category with objects that do not belong to it, and so they do not concern a change in the general ability to compare certain properties (Furmanski, Engel 2000). Furthermore, the above results have been replicated using various experimental designs (see de Leeuw et al. 2016; Goldston, Hendrickson 2010 for reviews). In consequence, such behavioral data provide a strong reason to believe that even the simplest forms of perceptual learning, which do not involve an obvious phenomenal change, involve a modification in represented lowerlevel properties.

A belief in occurrences of primitive categorization may also be motivated by an observation that it is sometimes difficult to conceptualize in virtue of which lower-level properties a given R-property is represented. A classic example concerns chicken sexers, who are able to recognize whether a chicken is male or female by touch, while it is difficult to 
explain the relevant differences (Biederman, Schiffrar 1987). However, even if chicken sexers are not able to precisely explicate the difference in lower-level properties connected with recognizing male and female chickens, they do not deny that such differences exist, because male and female chickens feel different when touched (males are described as 'round' and females as 'pointy', Biederman, Shiffrar 1987: 641). This suggests that alternative perceptual recognitions happen as a result of representing differing lower-level tactile properties connected with shape, size, or elasticity.

\subsection{Propositional factors}

According to the second option, the represented R-properties may change independently of changes in lower-level properties as a result of processing some propositional data. For instance, let's consider that one has an eccentric aunt who possesses a canary as well as its exact, advanced robotic replica. A person knows that their aunt always listens to the biological canary in the morning and the robo-canary in the evening. Because the person possesses this propositional knowledge, when they see her listening to a canary in the morning, the canary is recognized as a biological canary, while in the evening it is recognized as a robo-canary. In such a case, it seems that despite representing the same lowerlevel properties, the observer is making two different, correct recognitions: in the morning a 'biological canary' concept is attributed to the perceived item, and in the evening a distinct 'robo-canary' concept is applied.

While these recognitions are clearly different, it is by no means obvious that they are two different perceptual recognitions. Earlier, I have characterized perceptual recognition as a recognition that is an output of high-level mechanisms of perceptual categorization that match patterns formed as a result of perceptual learning with the current stimuli. However, if such a characterization is accepted, then it is unlikely that recognitions of a 'biological canary' and of a 'robo-canary' are two different perceptual recognitions.

First, the 'biological canary' and 'robo-canary' concepts are not formed solely as a result of perceptual learning, as their contents describe features concerning the internal structure of objects, which are not accessible to perceptual mechanisms. Second, the application of such a concept does not seem to consist in matching content with perceptual input, as the input does not contain any data concerning the properties relevant for identifying differences between the biological or artificial status of the perceived object. In consequence, it is unlikely that recognitions of 'biological' and 'robo-' canaries are the outputs of 
perceptual mechanisms, and so are not examples of perceptual recognitions. In fact, it seems that apart from making 'biological canary' and 'robo-canary' recognitions, in both situations described above, perceptual categorization mechanisms match the input, with the same canary-pattern formed as a result of earlier perceptual encounters with canaries. Thus, in both cases the observer makes the same perceptual recognition, and so applies the same Rproperty, which is accompanied, depending on the time of the day, by one of two alternative non-perceptual recognitions.

An analogous observation can be made when transpersonal cases are considered. According to biological data, aphids are able to reproduce both sexually and asexually. Let's imagine two people, Bob and Sally, who are able to recognize aphids. However, Bob only knows about aphids' sexual reproduction and Sally only about their abilities to reproduce asexually. Because of that, when seeing an aphid, they recognize it using different concepts, e.g. 'aphid-sexually-reproducing-insect' and 'aphid-asexually-reproducing-insect.' Because of the actual status of aphids, both of these recognitions can be treated as correct. However, analogously to the canaries described above, these do not seem to be different perceptual recognitions. It is so because the concepts of 'aphid-sexually-reproducing-insect' and 'aphidasexually-reproducing-insect' were not formed merely as a result of perceptual learning, and they cannot be applied to an object simply by matching their content with the perceptual input. To apply such concepts, some non-perceptual mechanisms must be engaged, which, for instance, are able to provide an inference between a certain look and a certain mode of reproduction. Of course, both Bob and Sally are likely to also make a genuine perceptual recognition when seeing an aphid. However, in doing so their perceptual mechanisms represent the same R-property by virtue of matching the input with the aphid-pattern formed as a result of similar processes of perceptual learning. ${ }^{4}$

\subsection{Temporal factors}

Another approach is to postulate that a represented R-property may change, despite the lack of lower-level changes, as a result of previous experiences. It is well-established that perceptual recognition can be affected by adaptation. For instance, after perceiving a picture of a male face, an androgynous face is more likely to be categorized as female (and analogous results have been obtained for ethnicity and emotional expression, see Fox, Barton 2006; Webster et al. 2004). Given this, we can imagine a situation in which one (a) perceives a male face that leads to adaptation, then (b) perceptually recognizes an androgynous face as female, 
after which one (c) perceives a female face that causes a different adaptation, and finally (d) perceptually recognizes the same androgynous face as male. It may seem that such adaptation-induced changes are examples of changing R-properties without modifying represented lower-level properties. However, in these cases, changes in acts of perceptual recognition are associated with modifying the sensitivity to various features (Webster et al. 2004: 560), additional feature grouping (Burge 2014), and involve changes in the perception of low-level, local properties like line curvature (Butler et al. 2007; Xu et al. 2008). This again suggests that each change in the attribution of R-properties is connected with a change in the way that lower-level properties are represented.

\subsection{High-level changes}

Finally, it should be noticed that in some situations it is plausible that R-properties supervene on other high-level properties. For instance, in the case of many artifacts, Rproperties may supervene on represented affordances, i.e. properties that describe the available ways of interacting with an object (Gibson 1986: 127-143, see Nanay 2011 for an interpretation within a representationalist framework). For example, it is likely that to represent that an object is a chair, it has to be represented such that one can sit on it. Other situations in which R-properties supervene on high-level properties may involve the perception of causal relations (Siegel 2009). We can imagine a case in which a person perceptually recognizes something as a dog running towards a wall. However, it does not stop before the obstacle but comes through it like a ghost. One may believe that the object will stop being recognized as a dog after such a non-standard causal pattern.

Nevertheless, while the above examples show that some R-properties may supervene on other high-level properties, they do not justify the claim that R-properties do not supervene on lower-level properties. This is because changes in other types of high-level properties are themselves connected with lower-level changes. Referring to the above examples, changes of affordances involve changes in the spatial properties of an object, and changes of casual properties are connected with changes in the spatiotemporal patterns of perceived events.

According to the above considerations, it is difficult to find changes in representing Rproperties that are not associated with changes in represented lower-level properties. Hence, while R-properties cannot be characterized as identical to lower-level properties, it is also unlikely that they are independent from them. It seems that the most plausible account of a 
relation between R-properties and lower-level properties is that R-properties supervene on lower-level properties, but not conversely.

\section{Perceptual kinds}

I have argued that R-properties, i.e. properties represented as result of acts of perceptual recognition, cannot be identified with lower-level properties, as for an R-property there is no single low-level property that is necessary and sufficient for representing this Rproperty. On the other hand, there is no evidence for the independence of R-properties from lower-level properties. Instead, R-properties seem to supervene on lower-level ones. Hence, R-properties may be understood as high-level ones, but are unlikely to be identical with usual natural kind properties, which are commonly independent from lower-level properties. Rproperties are not only high-level properties but they are also kind properties. This is because they are sortal properties that determine the identity conditions of objects. Overall, the properties represented as a result of perceptual recognition are sortal properties supervening on lower-level properties.

This result may be surprising, as it means that there are high-level, kind properties which differ from natural kinds, and which may be called 'perceptual kinds', that are represented as a result of perceptual recognition. In this context, we may ask what perceptual kinds are and what their function is. A plausible explanation can be formulated relying on the fact that the perceived properties of objects are not distributed at random, but form patterns and regularities. For instance, it is unlikely that something with a shape of an elephant's trunk is attached to something with a chicken-shaped body, but it is very likely that something with a beak will also have skin that is covered with feathers. The perceptual system learns such regularities and relying on them categorizes objects as belonging to perceptual kinds. On the one hand, perceptual kinds have to be constructed by relying on lower-level properties, and in fact they supervene on lower-level properties. However, they cannot be identified with conjunctions of lower-level properties as it is likely that their logical structures contain disjunctions as well as modal elements expressing the lower-level properties that are possible or probable for an object of a given perceptual kind.

It seems clear that perceptual kinds based on learned regularities can serve useful functions within a cognitive system. First, they allow us to predict, without engaging postperceptual mechanisms, currently inaccessible properties of an object given the available input. Second, perceptual kinds help in re-identifying objects despite changes in perceived 
properties, as they determine the changes that are essential. Third, they support the higher mechanisms of cognitive recognition, because classifying an object as an exemplar of a perceptual kind usually helps to determine the natural kind property that should be attributed to it in thought. Overall, it seems that the arguments developed within the paper distinguish a class of high-level, kind properties that play an important role in perceptual systems which are able to learn regularities between lower-level perceivable properties, while not having access to deep structures that define usual natural kinds.

\section{Acknowledgements}

The author would like to thank participants of SemDok seminar at Jagiellonian University, participants of workshop 'Naturalistic Approaches to Content and Consciousness' at Polish Academy of Sciences, participants of spring school 'Rethinking the Senses' organized by University of Warwick, participants of 10th Cracow Cognitive Science Conference at Jagiellonian University, and two anonymous reviewers for their comments concerning the earlier versions of the paper. The work was supported by the National Science Center (Poland) Grant 2016/20/S/HS1/00090.

\section{References}

Bayne, T. (2009). 'Perception and the Reach of Phenomenal Content', The Philosophical Quarterly 59(236), pp. 385-404.

Bayne, T. (2016). 'Gist!', Proceedings of the Aristotelian Society 116(2), pp. 107-126.

Biederman, I., Shiffrar, M. M. (1987). 'Sexing Day-Old Chicks: A Case Study and Expert Systems Analysis of a Difficult Perceptual Learning Task', Journal of Experimental Psychology: Learning, Memory, and Cognition 13(4), pp. 640-645.

Block, N. (2014). 'Seeing-As in the Light of Vision Science', Philosophy and Phenomenological Research 89(1), pp. 560-572.

Briscoe, R. (2015). 'Cognitive Penetration and the Reach of Phenomenal Content', in J. Zeimbekis and A. Raftopoulos (eds.) The Cognitive Penetrability of Perception. Oxford: Oxford University Press, pp. 174-199.

Brogaard, B. (2013a). 'Do We Perceive Natural Kinds Properties?', Philosophical Studies 162, pp. 35-42.

Brogaard, B. (2013b). 'Phenomenal Seemings and Sensible Dogmatism', in C. Tucker (ed.) Seemings and Justification. Oxford: Oxford University Press, pp. 270-289. 
Brogaard, B. and Chomanski, B. (2015). 'Cognitive Penetrability and High-Level Properties in Perception: Unrelated Phenomena?', Pacific Philosophical Quarterly 96(4), pp. 469-486.

Bullot, N. J. and Rysiew, P. (2007). 'A Study in the Cognition of Individuals' Identity: Solving the Problem of Singular Cognition in Object and Agent Tracking', Consciousness and Cognition 16, pp. 276-293.

Burge, T. (2014). 'Reply to Block: Adaptation and the Upper Border of Perception', Philosophy and Phenomenal Research 89(1), pp. 573-583.

Butler, A., Oruc, I., Fox, Ch. J. and Barton, J. J. S. (2007). 'Factors Contributing to the Adaptation Aftereffects of Facial Expression', Brain Research 1191, pp. 116-126.

Carey, S. and Xu, F. (1999). 'Sortals and Kinds. An Appreciation of John Macnamara', in R. Jackendoff, P. Bloom and K. Wynn (eds.) Language, Logic, and Concepts. Cambridge, MA: The MIT Press, pp. 311-336.

Carey, S. and Xu, F. (2001). 'Infants' Knowledge of Objects: Beyond Object Files and Object Tracking', Cognition 80, pp. 179-213.

Cavedon-Taylor, D. (2015). 'Kind Properties and the Metaphysics of Perception: Towards Impure Relationalism', Pacific Philosophical Quarterly 96(4), pp. 487-509.

Connolly, K. (2014). 'Perceptual Learning and the Contents of Perception', Erkenntnis 79(6), pp. 1407-1418.

Drew, T., Horowitz, T. S. and Vogel, E. K. (2013). 'Swapping Or Dropping? Electrophysiological Measures of Difficulty During Multiple Object Tracking', Cognition 126, pp. 213-223.

Edelman, S. and Bülthoff, H. H. (1992). 'Orientation Dependence in the Recognition of Familiar and Novel Views of Three-Dimensional Objects', Vision Research, 32(12), pp. 2385-2400.

Fenske, M. J., Aminoff, E., Gronau, N. and Mar, M. (2006). 'Top-Down Facilitation of Visual Object Recognition: Object-Based and Context-Based Contributions', Progress in Brain Research 155, pp. 3-21.

Fish, W. (2013). 'High-Level Properties and Visual Experience', Philosophical Studies 162, pp. 43-55.

Fox, Ch. J. and Barton J. J.S. (2006). 'What is Adapted in Face Adaptation? The Neural Representations of Expressions in the Human Visual System', Brain Research 1127, pp. 80-89.

Furmanski, S. C. and Engel, S. A. (2000). 'Perceptual Learning in Object Recognition: Object Specificity and Size Invariance', Vision Research 40, pp. 473-484. 
Gibson, J.J. (1986). The Ecological Approach to Visual Perception. New York: Taylor \& Francis.

Goldstone, R. (1994). 'Influences of Categorization on Perceptual Discrimination', Journal of Experimental Psychology: General 123(2), pp. 178-200.

Goldstone, R. L. and Hendrickson, A. T. (2010). 'Categorical Perception'. WIREs Cognitive Science 1, pp. 69-78.

Gordon, R. D. and Irwin, D. E. (1996). 'What's in an Object File? Evidence from Priming Studies', Perception \& Psychophysics 58(8), pp. 1260-1277.

Gordon, R. D. and Irwin, D. E. (2000). 'The Role of Physical and Conceptual Properties in Preserving Object Continuity', Journal of Experimental Psychology: Learning, Memory, and Cognition 26(1), pp. 136-150.

Hummel, J. E. (2013). 'Object Recognition'. in D. Reisburg (ed.) Oxford Handbook of Cognitive Psychology. Oxford: Oxford University Press, pp. 32-46.

Hummel J. E. and Biederman I. (1992). 'Dynamic Binding in a Neural Network for Shape Recognition', Psychological Review 99(3), pp. 480-517.

Jagnow, E. (2015). 'Can We See Natural Kinds Properties?', Epistemology \& Philosophy of Science 44(2), pp. 183-205.

Kahneman, D., Treisman, A. M. and Gibbs, B. J. (1992). 'The Reviewing of Object Files: Object-Specific Integration of Information', Cognitive Psychology 24(2), pp. 175-219.

de Leeuw, J. R., Andrews, J. K., Livingston, K. R. and Chin, B. M. (2016). 'The Effects of Categorization on Perceptual Judgment are Robust across Different Assessment Tasks', Collabra 2(1), 9.

Lyons, J. (2005a). 'Clades, Capgras, and Perceptual Kinds', Philosophical Topics 33(1), pp. 185-206.

Lyons, J. (2005b). 'Perceptual Beliefs and Nonexperiential Looks'. Philosophical Perspectives 19, pp. 237-256.

MacClelland, T. (2016). 'Gappines and the Case for Liberalism about Phenomenal Properties'. The Philosophical Quarterly 66(264), pp. 536-558.

Matthen, M. P. (2004). 'Features, Places, and Things: Reflections on Austen Clarks's Theory of Sentience', Philosophical Psychology 17(4), pp. 497-518.

Matthen, M. (2005). Seeing, Doing, and Knowing. A Philosophical Theory of Sense Perception. Oxford: Oxford University Press.

Moore, C. M., Stephens, T. and Hein, E. (2010). 'Features, as well as Space and Time, Guide Object Persistence', Psychonomic Bulletin \& Review 17(5), pp. 731-736. 
Nakayama, K., He Z. J. and Shimojo, S. (1995). 'Visual Surface Representation: A Critical Link between Lower-level and Higher-level Vision', in S. M. Kosslyn and D. N. Osherson (eds.) Vision: In Invitation to Cognitive Science. Cambridge, MA: The MIT Press, pp. 1-70.

Nanay, B. (2011). 'Do We See Apples as Edible?', Pacific Philosophical Quarterly 92, pp. 305-322.

Oksama, L. and Hyona, J. (2008). 'Dynamic Binding of Identity and Location Information: A Serial Model of Multiple Identity Tracking', Cognitive Psychology 56, pp. 237-283.

Pacherie, E. (2000). 'Levels of Perceptual Content', Philosophical Studies 100, pp. 237-254.

Price, R. (2009). 'Aspect-Switching and Visual Phenomenal Character', The Philosophical Quarterly 59(236), pp. 508-518.

Prinz, J. (2007). 'The Intermediate-Level Theory of Consciousness', in M. Velmans and S. Schneider (eds.) The Blackwell Companion to Consciousness. Malden: Blackwell Publishing, pp. 247-260.

Prinz, J. (2013). 'Siegel's Get Rich Quick Scheme', Philosophical Studies 163, pp. 827-835.

Pylyshyn, Z. W. (2007). Things and Places. How the Mind Connects with the World. Cambridge, MA: MIT Press.

Ramachandran, V. S, Armel, C. Foster, C. and Stoddard, R. (1998). 'Object Recognition Can Drive Motion Perception', Nature 395, pp. 852-853.

Reiland, I. (2014). 'On Experiencing High-Level Properties', American Philosophical Quarterly 51, pp. 177-187.

Reiland, I. (2015). 'Expereince, Seemings, and Evidence', Pacific Philosophical Quarterly 96, pp. 510-534.

Riddoch, M. J. and Humphreys, G. W. (1987). 'A Case of Integrative Visual Agnosia', Brain 110, pp. 1431-1462.

Schyns, P. G. and Murphy, G. L. (1994). 'The Ontogeny of Part Representation in Object Concepts', The Psychology of Learning and Motivation 31, pp. 301-349.

Schyns, P. G. and Rodet, L. (1997). 'Categorization Creates Functional Features', Journal of Experimental Psychology: Learning, Memory, and Cognition 23(3), pp. 681-696.

Shiffrar, M. and Freyd, J. J. (1990). 'Apparent Motion of the Human Body', Psychological Science 1(4), pp. 257-264.

Siegel, S. (2006). 'Which Properties are Represented in Perception', in T. S. Gendler and J. Hawthorne (eds.) Perceptual Experience. Oxford: Oxford University Press, pp. 481-503.

Siegel, S. (2009). 'The Visual Experience of Causation', The Philosophical Quarterly 59(236), pp. 519-540. 
Silins, N. (2013). 'The Significance of High-Level Content', Philosophical Studies 162, pp. $13-33$.

Solberg, J. L. and Brown, J. M. (2002). 'Recognition Affects the Perception of Apparent Motion', Journal of Vision 2, p. 678.

Ullman S. (1996). High-Level Vision. Object Recognition and Visual Cognition. Cambridge, MA: The MIT Press.

Van De Walle, G. A., Carey, S. and Prevor, M. (2000). 'Bases for Object Individuation in Infancy: Evidence from Manual Search', Journal of Cognition and Development 1(3), pp. 249-280.

Wei, L., Zhang, X., Lyu, C. and Li, Z. (2016). 'The Categorical Distinction Between Targets and Distractors Facilitates Tracking in Multiple Identity Tracking Task', Frontiers in Psychology 7, 589.

Webster, A. M., Kaping, D., Mizokami, Y. and Duhamel, P. (2004). 'Adaptation to Natural Facial Categories', Nature 428, pp. 557-561.

Wiggins, D. (2001). Sameness and Substance Renewed. Cambridge: Cambridge University Press.

$\mathrm{Xu}, \mathrm{F}$. (2007). 'Sortal Concepts, Object Individuation, and Language', TRENDS in Cognitive Science 11(9), pp. 402-406.

$\mathrm{Xu}$, F. and Carey, S. (1996). 'Infants' metaphysics: The Case of Numerical Identity', Cognitive Psychology 30, pp. 111-153.

$\mathrm{Xu}$, F., Carey, S. and Quint, N. (2004). 'The Emergence of Kind-based Object Individuation in Infancy', Cognitive Psychology 49, pp. 155-190.

Xu, H., Dayan, P., Lipkin, R. M. and Quian, N. (2008). 'Adaptation across the Cortical Hierarchy: Low-Level Curve Adaptation Affects High-Level Expression Judgements', The Journal of Neuroscience 28(13), pp. 3374-3383.

Institute of Philosophy and Sociology, Polish Academy of Sciences, ul. Nowy Swiat 72, 00330 Warsaw, Poland

\footnotetext{
${ }^{1}$ According to Reiland, quasi-sensory/quasi-cognitive phenomenology is a phenomenology of 'seemings' which are passive as sensory representations, but conceptual as cognitive representations (e.g., judgements).

${ }^{2}$ In the debates concerning perceptual content there is no widely-accepted, precise criterion allowing to decide which properties are lower-level. I do not attempt to resolve this issue and I simply use the term 'lower-level
} 
properties' to name those properties which serve as common examples of lower-level properties in philosophical papers.

${ }^{3}$ Sometimes the term 'sortal properties' is used as a synonym of natural kind properties (e.g., Nanay 2011). In this paper, I use it in a weaker sense merely to distinguish those properties that determine identity conditions of objects.

${ }^{4}$ Significantly different histories of perceptual learning would cause Bob and Sally to have distinct aphidpatterns. This would mean that if they apply their patterns to the same aphid-input (a) one of them makes a misrecognition by applying his or her pattern, or (b) that in fact each person represents different lower-level properties because distinct learning histories have resulted in varying sensitivity to some of the object's features (see section 4.1). 